Verivey, W. F., and Williams, H. R., Jr. (1963), Pp. 476-483 in Antimicrobial Agents and Chemotherapy-1962. Ann Arbor: Braun-Brumfield, Inc.

White, A., and Varga, D. (1961), Arch. intern. Med., 108, 671 .
Whitehouse, A. C., Morgan, J. G., Schumacher, JK and Hamburger, M. (1963), Pp. 304-392 in Ant microbial Agents and Chemotherapy-1962. Ann Arbor: Braun-Brumfield, Inc.

Wilson, R., and HAMBurger, M. (1957), Amer. Med., 22, 437.

\title{
PENICILLIN THERAPY IN RELATION TO CHEST DISEASES
}

\author{
C. A. Green \\ Department of Microbiology \\ The Royal Victoria Infirmary and the University, Newcastle-upon-Tyne.
}

THE term chest infection is a wide one and in one sense there is no need to discuss penicillin therapy in relation to chest infection. Thus classical lobar pneumonia is a rarity in hospital in these days and is regarded by the present generation of medical students as a clinical curio. The clinical bacteriologist in his laboratory has come to regard colonies of Streptococcus pneumoniae as being of relatively little importance, since he knows full well that by the time he has cultured the organism, the clinician will have treated the patient with some form of penicillin and that in all probability, the clinical condition will have resolved. So much so that an organism alleged to be a pneumococcus which is resistant to penicillin would be regarded with suspicion and in fact it is almost unknown in our laboratory to find such an organism. Thus therapy for conditions such as lobar pneumonia presents no problem. Penicillin $G$ or one of its analogues is given and rarely does the condition progress to the classical picture.

Ours is a general hospital which also functions as the teaching hospital for the University of Newcastle-upon-Tyne. Since 1950 we have made it our standard practice to examine the sensitivity of all pathogens isolated, to a reasonably wide range of antibiotics. From our records Dr. A. J. Wort has constructed two tables. In late 1950 and 1951 we did not record the diagnoses or sources of the organisms which we isolated and the original requests inevitably are no longer available.

We are not, therefore, able to show the pattern of sensitivity at this time in relation to chest infection. I thought it, however, fair to make certain deductions from the available data. Table I shows that in the winter period 1950-1951 we isolated a total of 232 staphylococci and of these $132(57 \%)$ werie designated penicillin resistant. I confess that am surprised by this because I would have thought that penicillin resistance had not be come a problem of this magnitude. Howeve? the comparative figure for a similar number of organisms studied in 1962 as part of our ear work with orbenin revealed an increase up to $73 \%$ penicillin $\mathrm{G}$ resistant strains.

More recently in 1957 we introduced hhe practice of recording in our sensitivity reegrd books the diagnoses and an indication of the source of the organisms which had been isolated. From that time we have been able to estimate the incidence of staphylococcal chest infections. Table II shows the pattern of even for the period, November-February inclusi for the past seven years. We have found that the post-operative chest infection, which commonly due to a hospital staphylococcus, is a serious problem and roughly $80 \%$ of these staphylococci are resistant to penicillin $\mathrm{G}$ : This, of course, is not to say that other organ isms do not on occasions become involved and in common with other workers we have found that Proteus spp. and Ps. pyocyanea are diffis cult to control. Nevertheless the staphylococcus is our chief problem since it appears to be se easily transferred from one patient to anothe and does so easily colonize the nasopharyn of both patients and staff alike. Many of the staphylococci which we have considered are resistant to a wide range of antibiotics, usually being sensitive only to erythromycin, novos biocin and chloramphenicol. Our clinical cob leagues are not very enthusiastic about using chloramphenicol and this effectively limits the choice of antibiotic. The release of methicillief in 1960 was, therefore, a real advance in therapy and it rapidly became the drug of 
TABLE I.

Incidence of Penicillin Resistance in all Staphylococci Isolated during Winter 1950-51 and 1962 (Feb. only)

\begin{tabular}{ccccc}
\hline Year & Total & Resistant & Sensitive & $\%$ Resistant \\
1951 & 232 & 132 & 100 & 57.0 \\
1962 & 235 & 172 & 63 & 72.8 \\
\hline
\end{tabular}

TABLE II.

Resistance Pattern of Staphylococci Isolated from Respiratory Tract Winter Period 1957-1964 Inclusive

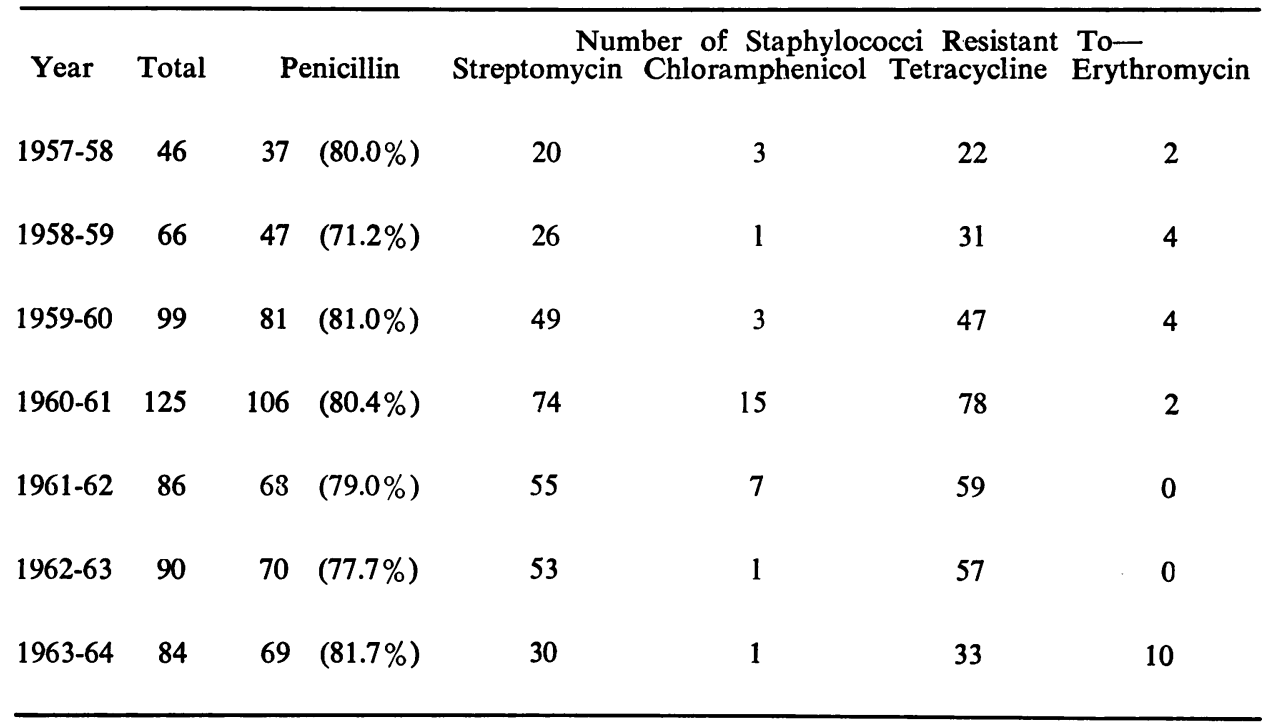

choice in dealing with this kind of infection. Its disadvantage was of course that it could only be given parenterally.

We were privileged to be among the first people to be given the opportunity of using the new isoxazolyl penicillins and since then cloxacillin, which was one of three drugs, subjected to clinical trial in April, 1962 (Stewart, 1962) has become the drug of choice in our hospital for the management of resistant staphylococcal infections.

In order to illustrate something of our results I thought it would be useful to present details of two cases-one is a failure - a patient who died in the early stage of therapy with cloxacillin. The other, by contrast, is a very successful one and I shall describe this case first. The patient concerned had had an aortic graft inserted in 1960 and two years later was returned to the same surgical clinic with an intestinal obstruction. He was operated upon and the obstruction was relieved. His convalescence was, however, very stormy. For the first three weeks he had an intermittent pyrexia and he was treated with tetracycline. On the evening of the twenty-first day in hospital he had high fever. He was immediately given penicillin $G$ and streptomycin by injection and sputum culture was undertaken and the following day we recovered a staphylococcus which was resistant to all the antibiotics except erythromycin, novobiocin, methicillin and cloxacillin. Therapy with cloxacillin was instituted and the effect was dramatic. His serum levels were estimated and we recovered what for us was the unique figure of $16 \mu \mathrm{g}$. throughout the whole period of study. These levels are very high by 
our standards and although we have looked for an explanation for this we have not so far found one. His temperature fell and he made good progress. He is still well at the present time. His X-rays formed a fascinating series because at the onset in this stage of his illness there were definite infective changes and there was a suggestion of cavity formation together with a fluid level. In other words he probably had a lung abscess which resolved completely during therapy without surgical intervention.

The second case is less encouraging but nevertheless informative. She had been admitted to hospital with pneumonia from which no organism was recovered. She was treated with sulphonamides and made reasonably satisfactory progress. Then she developed a lesion in the left side which was thought to be a perinephric abscess. At operation this diagnosis was found to be incorrect but there was a large collection of pus above the diaphragm on the left side. Surgical drainage was carried out and a resistant staphylococcus was recovered from the pus and shortly afterwards from her sputum. This was found to be sensitive to cloxacillin and although she was very ill therapy was commenced immediately using the parenteral preparation. She made very little response and died thirty hours after therapy had been instituted.

Post mortem showed extensive abscess formation in the lungs and from the centre of these abscesses, staphylococci of identical sensitivity pattern and phage type were recovered. It had not been possible to estimate a serum level in this patient but I do think that this is the crux of the whole matter. Older workers will remember that this question has been hotly debated from time to time with various conditions, S.B.E., osteitis and the like. Two factors seem to me to be important:-

1. The nature of the lesion. This I think is an important factor in this case. In the centre of the larger abscess are collections of Gram positive organisms surrounded by an enormous collection of inflammatory cells and fibrin. It may well be that the ability of the staphylococcus to produce coagulase is an important factor in the production of this reaction. The net result of this is to produce a completely avascular mass of tissue through which antibiotics penetrate only with difficulty. If the serum levels are low or intermittent the concentration at the centre of such a mass will be very low and in fact will not reach very effective levels. In order to be effective, the antibiotic must penetrate to the centre of the lesion and, therefore, the levels in the surround $-\frac{\mathbb{D}}{\circ}$ ing tissues must be high and must remain high for long periods in order that the gradient of any diffusion which takes place will be towards? the centre of the lesion and not away from it.

2. A second factor is that the continued $\frac{\bar{m}}{\frac{1}{2}}$ multiplication of the staphylococci in the centre $\stackrel{\mathbb{\Phi}}{\Omega}$ of such a lesion may produce large quantitieso of exotoxin. Certainly, in this particular patient at the time of her death there was a good deal of evidence to suggest that she was suffering $\vec{\omega}$ from a profound toxæmia. I, therefore, favouro the maintenance of high and, if at all possibleco continuous levels in these conditions all of which are potentially fatal.

One régime by which this would be possible is to give the drugs by injection and wheno clinical recovery has commenced to give orait therapy in adequate doses thereafter. I person-o ally take the view that $500 \mathrm{mgm}$. is a suitable adult dose for cloxacillin and that if necessaryo this can be given four hourly. Our studies on serum blood levels have shown that this dose régime does maintain adequate serum leve्cs: throughout the whole period.

Our pædiatric colleagues use this regime the management of some of their cases osteitis. There are possible objections to thise régime since parenteral therapy of this intensity could hardly be popular with the patient. AD third possibility and I think a good one is too give probenecid in addition to oral cloxacillin.

You will have noticed that I have con $\frac{1}{3}$ centrated almost entirely on the post-operative chest. This is very largely, of course, reflecting. the number of cases that we have seen and the extent of the problem as we see it.

We have, however, had a little experience with a problem which I am sure you have all? met before. Our pædiatric colleagues have had in their care a family in whom several of the? children are suffering from fibrocystic disease $>$ Two of these have acquired hospital staphylococci which in addition to the usuan staphylococcal backing have become resistant to fucidin. On the whole we have foundo cloxacillin the most useful drug in the manage- $\omega$ ment of these two patients.

\section{REFERENCE}

Stewart, G. T., et al. (1962): Report from six hospitals, Lancet, ii, 634. 